\title{
Sedimentation in flocculating colloidal suspensions
}

\author{
Jeffrey S. Abel and Gregory C. Stangle \\ School of Ceramic Engineering and Sciences, New York State College of Ceramics at Alfred University, \\ Alfred, New York 14802
}

Christopher H. Schilling

Department of Materials Science and Engineering, Iowa State University, Ames, Iowa 50011

Ilhan A. Aksay

Department of Chemical Engineering and Princeton Materials Institute, Princeton University, Princeton, New Jersey 08544

(Received 29 March 1993; accepted 17 September 1993)

\begin{abstract}
A combined experimental and theoretical investigation of the sedimentation of unstable colloidal ceramic suspensions has been performed. Suspensions containing submicron-sized $\alpha-\mathrm{Al}_{2} \mathrm{O}_{3}$ particles were prepared at various $\mathrm{pH}$ values in order to modify suspension stability. Particle volume fraction during sedimentation was determined as a function of position and time by gamma-ray densitometry. A population balance model was developed to account for various coagulation and decoagulation mechanisms that affect sedimentation behavior in flocculating suspensions. Model predictions were then compared with experimental measurements, in order to establish the validity of the theoretical model.
\end{abstract}

\section{INTRODUCTION}

Colloidal consolidation is the central physical process that occurs in a number of shape-forming processes (e.g., slipcasting and pressure filtration). ${ }^{1}$ The sedimentation-based process typically begins with a relatively dilute dispersion of fluid-suspended solid particles. The usual goal of such a sedimentation-based shape-forming process is to produce high-green density compacts. A sedimentation-based process is rather simple to describe quantitatively (and thus control) when the suspension is dilute and the individual particles are discrete. ${ }^{2}$ More concentrated suspensions that form clusters (or "agglomerates" or "flocs" or "aggregates") are much more difficult to describe quantitatively. ${ }^{3,4}$ Further, and perhaps more importantly from a practical viewpoint, concentrated, flocculating suspensions usually form lower density green bodies-that ultimately lead to severely flawed finished ceramic articles. ${ }^{5-7} \mathrm{~A}$ better understanding of the evolution of colloidal structures in ceramic shape-forming processes will lead to better control of the microstructure of the article, a reduction in the number of defects, etc.

Several studies of sedimentation and flocculation have been reported in the literature. For example, Batchelor ${ }^{8}$ began with the simplest system: a dispersed, dilute suspension containing particles of only one size. This early work was extended to consider (i) dispersed, dilute, and polydisperse suspensions, ${ }^{9-12}$ and (ii) dispersed, concentrated, and monodisperse suspensions. ${ }^{3}$ Similarly, flocculation has been treated by several authors, ${ }^{13-18}$ for quiescent and shear flow fields, and for
2- and N-particle systems. Simultaneous treatment of sedimentation and flocculation has, for all of its practical importance, been reported much less frequently.,2,19 Auzerais et al..$^{2,20}$ for example, studied sedimentation in an idealized flocculating suspension (that is, with a hardsphere or nearly hard-sphere interparticle potential), both experimentally and theoretically. Buscall ${ }^{21}$ has compiled a bibliography and review of the theoretical aspects of this area.

The literature provides only a few reports ${ }^{21}$ of flocculating sedimentation in which nondilute suspensions that contain more commonly encountered pair-potentials are considered. What is needed, of course, is a quantitative understanding of each of the physical and chemical processes that determine the behavior of sedimenting and flocculating particles and clusters. It is also important (as will be shown) to account for certain "declustering" (or decoagulation) processes that affect the overall sedimentation behavior of a particle-containing suspension. Ultimately, this can enable one to follow the microstructural evolution of such a suspension. This in turn has been shown to affect the success of each subsequent step in the ceramics fabrication process: drying, binder removal, and firing. Figure 1 illustrates this point well: slipcast $\mathrm{Al}_{2} \mathrm{O}_{3}$ using $\mathrm{HCl}$ and $\mathrm{NH}_{4} \mathrm{OH}$ as additives to adjust the pair potential. ${ }^{22}$

In this work, we have developed a theoretical model that describes flocculating sedimentation, such that particle cluster formation is accounted for. The model was then used to conduct computer experiments, in order to predict particle concentration as functions of both 


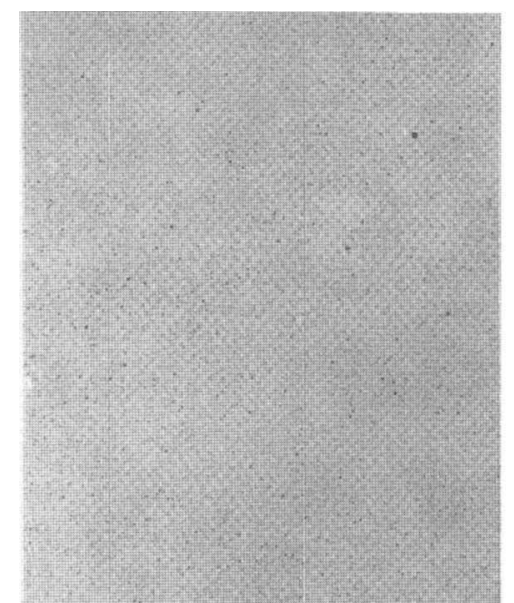

(a)
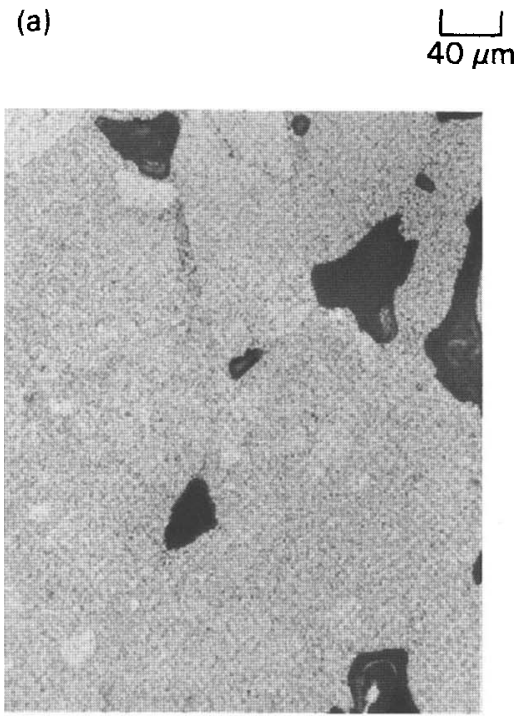

(d)

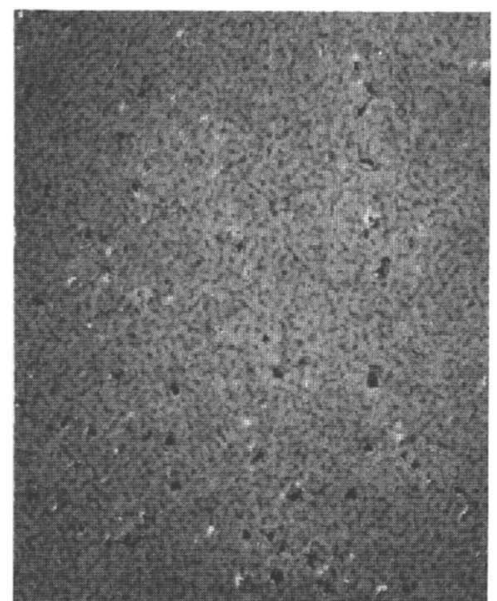

(b)

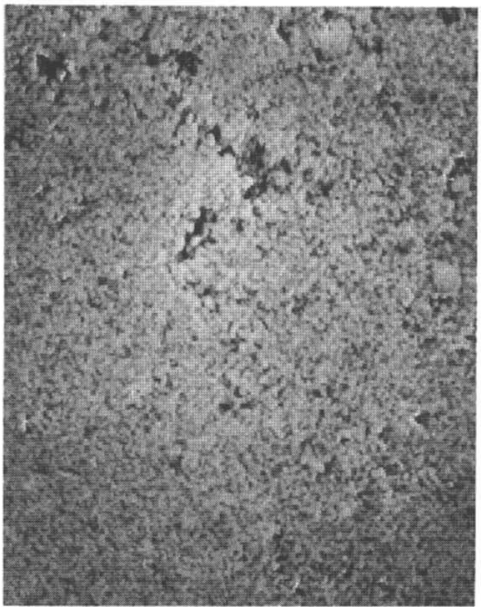

(e)
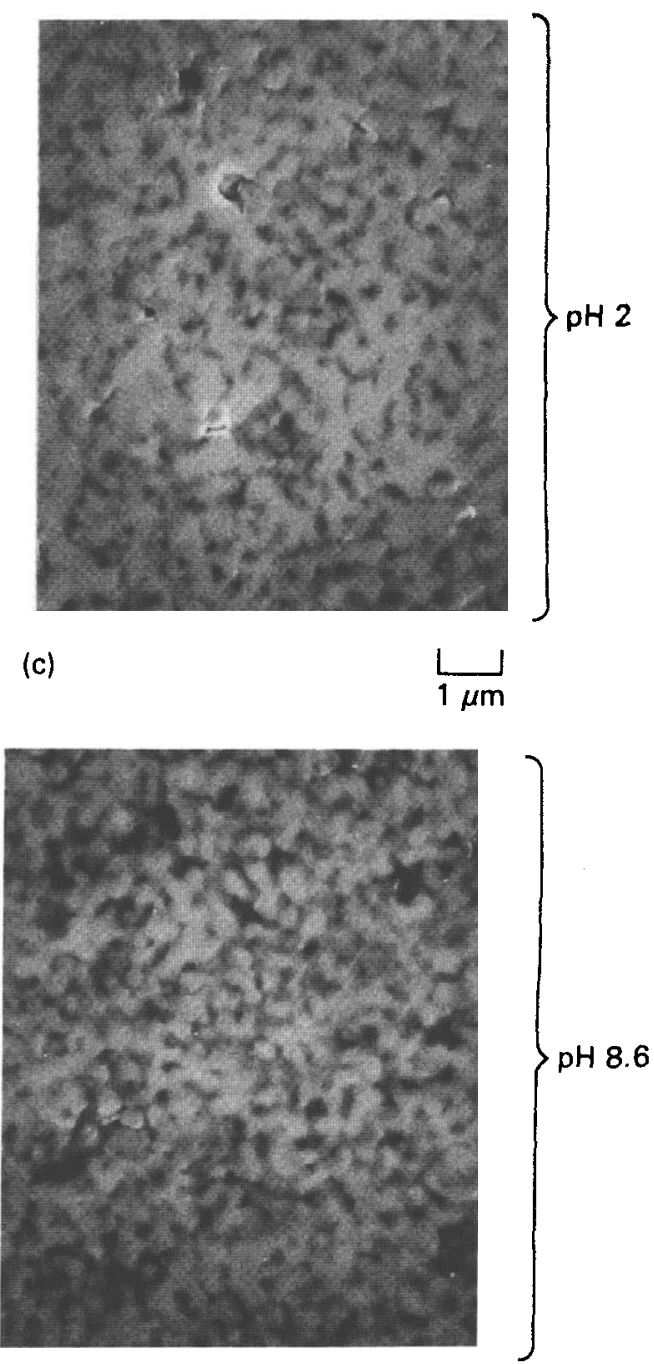

(f)

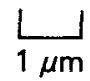

FIG. 1. Hierarchically sized pores in sintered alumina sediments. ${ }^{22}(\mathrm{a}-\mathrm{c})$ At $\mathrm{pH}=2$, repulsive surface forces produce dense-packed microstructures exhibiting first- and second-generation pores (approximately 0.1 and $0.5 \mu \mathrm{m}$, respectively). (d-f) At pH $=8.6$, attractive surface forces and the settling of agglomerates result in pores that exhibit multiple generations of size.

position and time. Corresponding experiments were then conducted to verify model predictions. The expected benefit to be derived from this work is to provide input for the development of an improved ceramics fabrication process, based on the detailed, improved understanding of the evolution of colloidal structures during consolidation.

\section{THEORETICAL MODEL}

\section{A. Physical description of the process}

Sedimentation is essentially a process in which density difference between constituent phases, and the imposition of an external field, cause differential movement of the phases. This results in the spatial segregation of the constituent phases. The sedimentation velocity of a single sphere in an infinite fluid settling at low Reynolds number is, of course, given by Stokes law. ${ }^{23}$ Stokes law becomes invalid, however, in more concentrated suspensions found in commercial and research settings; the processing of minerals, the processing of colloidal ceramics, and the treatment of industrial and municipal waste streams are some of the more important examples. In these cases, interactions between particles modify the settling behavior of a given particle, relative to that of an isolated particle. The most significant interactions are (i) hindered settling, (ii) clustering, and (iii) diffusion.

Hindered settling, as the term suggests, indicates that a particle's sedimentation velocity is decreased in the presence of other particles. That is, as the volume fraction of the dispersed phase is increased, the average separation distance between each particle of the discrete 
phase is decreased. Hydrodynamic interactions are quite strong at these short separation distances, and thus may retard the motion of a given particle. Sedimentation rate is therefore decreased. Figure 2(a) shows this schematically.

Clustering, on the other hand, can actually increase the sedimentation rate above that of a single particle. Particles may join to form clusters (under certain conditions). The clusters, having a larger radius, will have a larger sedimentation velocity in a given applied field when compared to that of a single particle. If the clustering process is reversible, however, a cluster may disassociate to form smaller clusters or particles; the latter will of course settle more slowly. See also Fig. 2(b).

Diffusion of particles and clusters is also important during a sedimentation process. As sedimentation proceeds, the solids concentration in the lower region of a sedimentation column is higher than that in the top. There is, in analogy with the better-known diffusion process involving solutes, a driving force for particles and clusters to move "down" the concentration gradient (or up the sedimentation column). As a result, there is an impetus for particles or clusters to resist sedimentation. The net effect is to reduce settling velocities of particles and clusters. This is shown in Fig. 2(c).

It was the purpose of this work to describe mathematically a sedimentation process involving solid spherical particles dispersed in a continuous liquid phase, in which hindered settling, cluster formation and breakup, and diffusion are explicitly accounted for. The approach used is that of the "population balance model," whereby the concentration of a cluster size " $j$ " (or " $j$-cluster," containing $j$ particles), at each spatial position and at all times, is modified by a variety of creation and loss mechanisms-corresponding, physically, to hindered settling, clustering, and diffusion.

\section{B. Population balance model}

The population balance approach ${ }^{24-27}$ has been employed in the study of various disperse systems, such as liquid droplets in a gas stream, gas bubbles in a liquid stream, liquid droplets in a second, continuous liquid, etc. The "population" of interest is the dispersed phase, where the overall population may be divided into various classes. The size, or number of elements, in each class may be important to monitor. The nature of the population is dynamic, since the number of classes and the number of elements in each class may change with time, as shown in Fig. 3. A properly constructed population balance model should account for (and predict) the makeup of the population as a function of time.

In the present model, a sedimentation column is divided into $\mathrm{N}$ slabs (Fig. 4). A sort of "subpopulation balance" is first performed in each slab, for all cluster sizes, as shown in Fig. 5. Four kinds of changes may

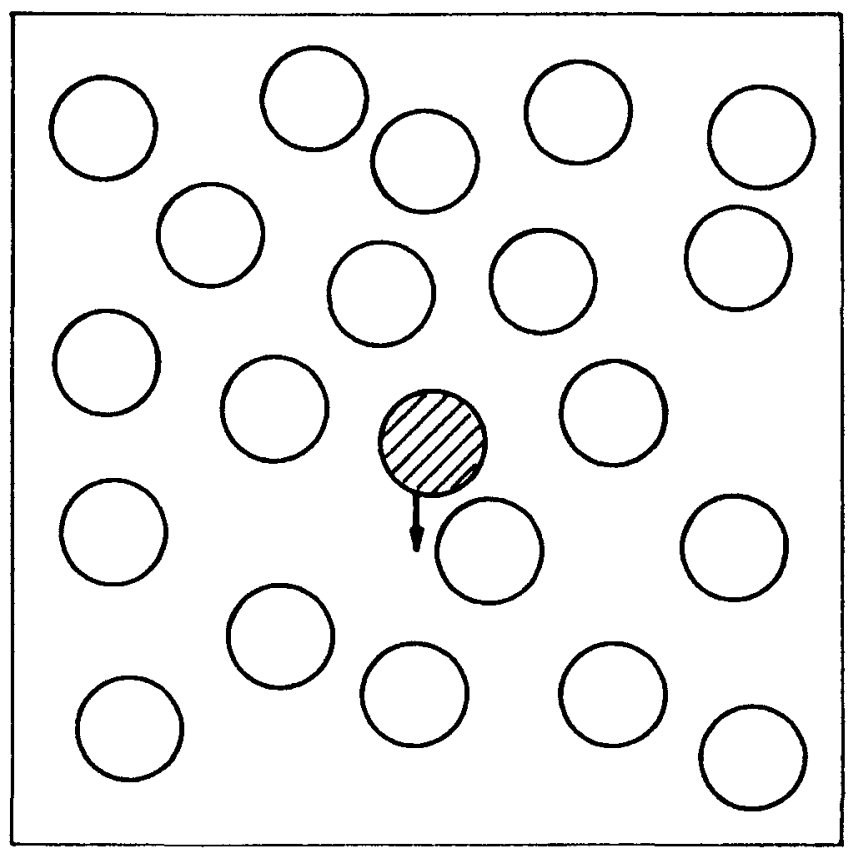

(a)

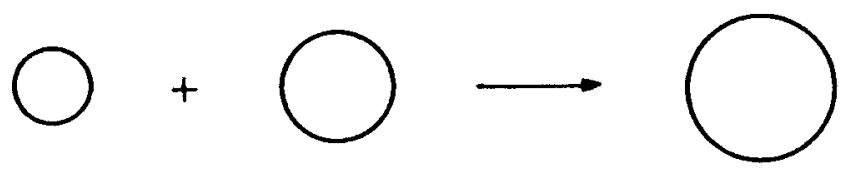

(b)

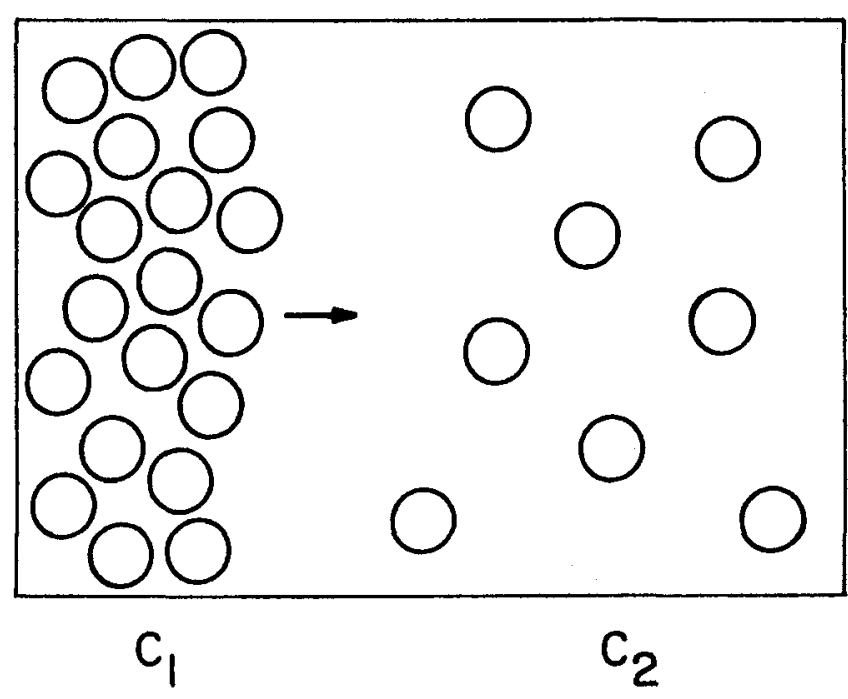

(c)

FIG. 2. Schematic representation of key clustering/declustering mechanisms that affect simple Stokesian sedimentation: (a) hindered settling, (b) cluster formation (or dissociation), and (c) diffusion (where $c_{1}>c_{2}$ ). 


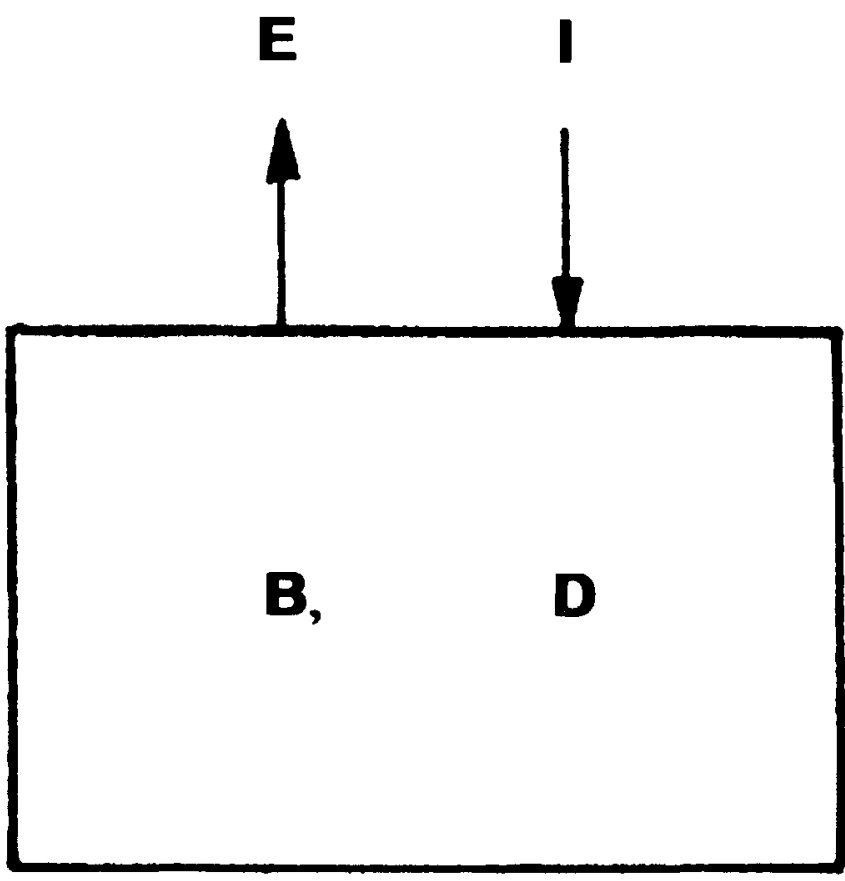

FIG. 3. General population balance model. $\mathrm{E}=$ emigration, $\mathrm{I}=$ immigration, $\mathrm{B}=$ birth, and $\mathrm{D}=$ death. $\mathrm{P}=\mathrm{P}_{0}+\mathrm{B}-\mathrm{D}+\mathrm{I}-\mathrm{E}$; $\mathrm{P}_{0}=$ initial population.

occur that can change the number of $j$-clusters in each slab. The first two are kinds of "immigration" and "emigration" processes, whereby $j$-clusters from neighboring slabs enter the slab of interest from, or $j$-clusters leave the slab of interest to, neighboring slabs, respectively. These correspond to the hindering settling and diffusion processes. The second two changes that may occur are "birth" and "death" processes. For example, a 3-cluster (or a cluster containing 3 particles) may be "born" when

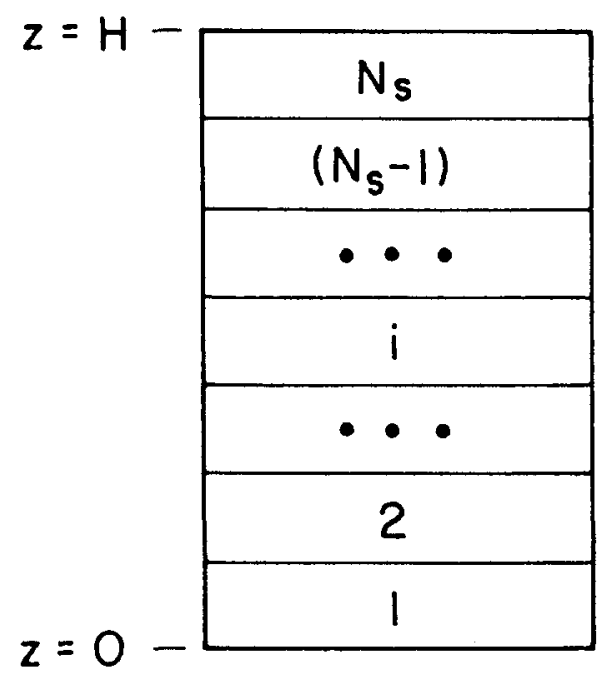

\section{lower $\epsilon$}

FIG. 4. Representation of sedimentation cell as used in population balance model calculations.

\section{higher $\epsilon$}

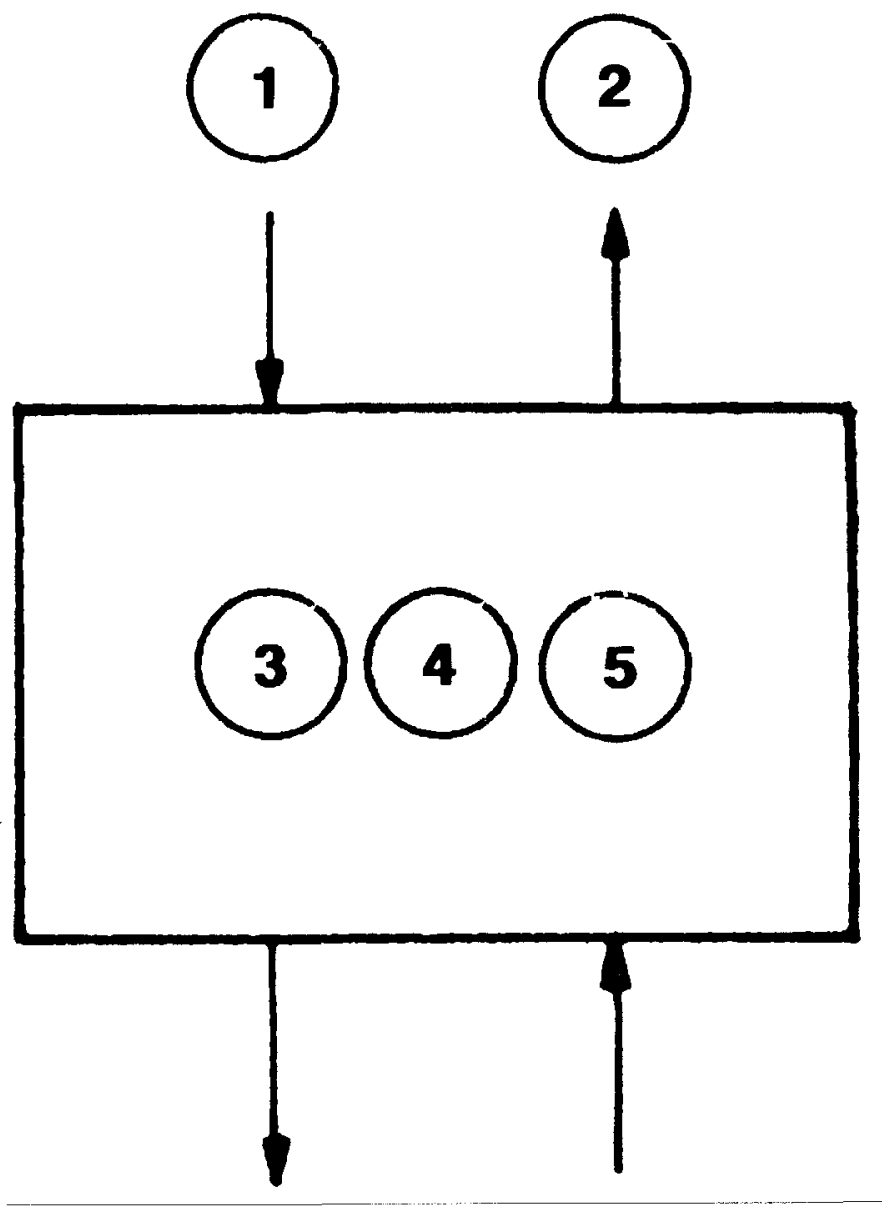

FIG. 5. Factors influencing the "subpopulation balance" in a single slab during sedimentation. (1) Settling (convection), (2) diffusion, (3) orthokinetic flocculation, (4) hydrodynamic-induced breakup, and (5) perikinetic flocculation.

a 1-cluster and a 2-cluster combine to form a 3-cluster. As this occurs, the "death" of a 1-cluster and a 2-cluster naturally takes place.

All of these processes may be described mathematically. Each description is given in more detail below.

\section{Immigration and emigration}

A slab may gain clusters by sedimentation and diffusion from neighboring slabs. A slab may also lose clusters to neighboring slabs by the same mechanisms. Sedimentation is given quite simply in terms of the convection flux of $k$-clusters into and out of the slab:

$$
J_{c, k}=-\frac{\partial\left(V_{k}^{\prime} C_{k}\right)}{\partial z},
$$


involving an incompressible liquid, the downward movement of solid particles or clusters requires a corresponding upflow of liquid. The upflow of liquid is given by:

$$
V^{\prime \prime}=\frac{\sum_{k} V_{k}^{\prime} C_{k} v_{k}}{1-\sum_{k} C_{k} v_{k}}
$$

where $V^{\prime \prime}$ is the velocity of the liquid with respect to the fixed (laboratory) reference frame, and $v_{k}$ is the volume of a $k$-cluster. The summations are carried out over all cluster sizes. Further, the sedimentation velocity of a $k$-cluster, relative to the surrounding fluid (which is itself nonstationary), is given by $V_{k}$ (Ref. 23):

$$
V_{k}=\frac{2\left(\Delta \rho^{*}\right) g r_{k}^{2}}{9 \eta^{*}}
$$

where $\Delta \rho^{*}$ is the effective difference in densities between the solid and liquid phases, $\eta^{*}$ is the effective fluid viscosity, $g$ is gravitational acceleration, and $r_{k}$ is the radius of a $k$-cluster:

$$
r_{k}=\left(\frac{3 k v_{1}}{4 \pi}\right)^{1 / 3}
$$

where $v_{1}$ is the volume of a "1-cluster" or primary particle. Equation (3) is a modified form of Stokes law for sedimentation, where $\Delta \rho^{*}$ and $\eta^{*}$ account for the nondilute nature of the suspension. In a given slab, then, the effective density difference is given here by:

$$
\Delta \rho^{*}=\Delta \rho^{*}(c)=\left(\rho_{s}-\rho_{L}\right)\left(1-\sum_{n} C_{n} v_{n}\right) .
$$

Equation (5) shows that the actual $\Delta \rho$ is modified by $\sum_{n} c_{n} v_{n}$, which is the total solids volume fraction in the slab. Similarly, the effective viscosity in a given slab is (Ref. 28):

$$
\eta^{*}=\eta_{0}\left(1+2.5 \phi+7.6 \phi^{2}+\ldots\right),
$$

where $\eta_{0}$ is the liquid viscosity, and [as in Eq. (6)] $\phi$ is given by:

$$
\phi=\sum_{n} C_{n} v_{n}
$$

Further, the diffusive flux of particles and clusters into and out of the slab is given by:

$$
J_{D, k}=\frac{\partial}{\partial z}\left(D_{k} \frac{\partial C_{k}}{\partial z}\right),
$$

where $D_{k}$ is the diffusion coefficient given by:

$$
D_{k}=\frac{\mathrm{k} T}{6 \pi \eta r_{k}}\left(1+1.56 \phi+0.91 \phi^{2}+\ldots\right)
$$

after Beenakker and Mazur. ${ }^{29}$ Note that $\mathrm{k}$ is Boltzmann's constant and $T$ is absolute temperature. This term became more important as $r_{k} \rightarrow 0$ and $\phi \gg 0$.

\section{Birth and death}

Clusters of a given size can be created or destroyed in a slab by either hydrodynamic or Brownian mechanisms. Each mechanism is treated separately.

\section{Hydrodynamic clustering and declustering}

Large clusters have a higher sedimentation velocity than small clusters. As a result, a large cluster may actually overtake a smaller cluster and, upon touching, flocculate. ${ }^{30}$ An $i$ - and $j$-cluster would form a $k$-cluster, where $k=(i+j)$. A population balance would show the birth of a $k$-cluster, and the death of one $i$-cluster and one $j$-cluster. A balance within a slab would show that the overall change in $k$-clusters must be influenced by the concentration of $i$ - and $j$-clusters, by the difference in settling velocities, and by the cross-sectional area of a cluster (the latter is by analogy with "scattering crosssectional area" in various scattering phenomena). The birth of $k$-clusters is then given by:

$$
B_{1, k}=\sum_{j=1}^{[k / 2]} C_{j} C_{k-j}\left|V_{j}-V_{k-j}\right| \pi\left(r_{j}+r_{k-j}\right)^{2} .
$$

Note that the summation index " $j$ " runs from 1 to $[k / 2]$, where $[k / 2]$ is the greatest integer less than or equal to $k / 2$ (e.g., if $k=4$, then $[k / 2]=2$; if $k=5$, then $[k / 2]=2$ ). Similarly, a $k$-cluster could be lost by combining with another cluster, of size $l$, say, to form a $(k+$ $l$ )-cluster. The death of a $k$-cluster would then occur:

$$
D_{1, k}=-\sum_{j=1}^{N_{s}-k} C_{k} C_{j}\left|V_{k}-V_{j}\right| \pi\left(r_{k}+r_{j}\right)^{2} .
$$

Comparison of Eqs. (10) and (11) shows that the signs on the right-hand sides are opposite, and that the summation ends in Eq. (11) at $\left(N_{s}-k\right)$, where $N_{s}$ is the largest cluster size considered. (This prevents formation of clusters larger than $N_{s}$.)

Alternatively, the drag around and through a cluster during sedimentation may cause a cluster to undergo fragmentation. ${ }^{30}$ This is typically considered to be a first-order rate process, and applies principally to very large clusters (often $>100 \mu \mathrm{m}$ ). For completeness, it is included here as:

$$
B_{2, k}=\sum_{m=k+1}^{N_{s}} k_{k, m-k}^{m} C_{m}\left(1+\delta_{k, m-k}\right) .
$$

Equation (12) shows that the summation runs over all clusters larger than $k$ and is proportional to the particle number density of each of these larger clusters. The rate constant, $k_{k, m-k}^{m}$, is given by:

$$
k_{k, m-k}^{m}=k(\Delta \rho) \frac{(m)(m !)}{(k !)(m-k) !}
$$


Similarly, the hydrodynamic-induced breakup of a $k$-cluster is similarly given by:

$$
D_{2, k}=-\sum_{j=1}^{[k / 2]} k_{j, k-j}^{k} C_{j},
$$

where all notation has been defined above. [It should be noted here that in Eq. (13), the product of factorials represents all of the ways in which the particles of an $m$-cluster can break down, or be partitioned, into two groups of size $k$ and size $(m-k)$.]

\section{Brownian clustering and declustering}

Small solid particles (with diameters below $\sim 10 \mu \mathrm{m}$ ) also experience significant random nonhydrodynamic displacements or Brownian motion. The magnitude of the random displacements increases as particle (or cluster) size decreases. There is a nonzero probability that two such clusters may collide and form a larger cluster ${ }^{30-32}$ Alternatively, there is also a nonzero probability that a cluster of a given size may break into two or more pieces as a result of a Brownian dissociation process. Now, Smoluchowski developed an expression for the collision frequency of hard-sphere Brownian particles (e.g., particles in a dispersion for which Brownian motion is significant). In this case, a rate equation is given as:

$$
\frac{d C}{d t}=-8 \pi D r C^{2}
$$

where $D$ is the Brownian diffusion coefficient, given as:

$$
D=\frac{\mathrm{k} T}{6 \pi \eta_{0} r}
$$

where $r$ is the particle radius, $C$ is the particle concentration (number density), and the other terms are as before. Note that Eq. (15) applies only to a monodisperse suspension of one-clusters undergoing coagulation, and only for hard-sphere nonhydrodynamic interactions. When the particles do experience other forms of nonhydrodynamic interactions (e.g., as described by DLVO, soft-sphere, Lennard-Jones, etc., interaction potentials ${ }^{31}$ ), the rate expression in Eq. (15) would need to be modified. That is, in a hard-sphere system, all "attempted" collisions would be successful, since the influence of one particle upon the motion of another would not be felt until the two collided. Spheres with a repulsive potential, for example, would undergo successful collisions less frequently than in the case of hard-sphere particles. In such a case, the right-hand side of Eq. (15) must be modified to account for only successful collisions. The stability ratio, $W$, or collision efficiency factor, may be defined $\mathrm{as}^{31}$ :

$$
W=\int_{2 r}^{\infty} \frac{1}{R^{2}} \exp \left(\frac{V}{\mathrm{k} T}\right) d R
$$

where $V$ is the interparticle interaction potential. The integration in Eq. (17) is performed from the particle surface to infinity. For a DLVO potential, $V$ is given by the expression:

$$
\begin{aligned}
V= & -\frac{A_{H}}{12}\left[\frac{1}{s^{2}-1}+\frac{1}{s^{2}}+2 \ln \left(\frac{r^{2}-1}{r^{2}}\right)\right] \\
& +\pi \epsilon(2 r) \phi_{0}^{2} \ln \left[1+\exp \left(\kappa^{*}\{s-1\}\right)\right]
\end{aligned}
$$

where $s=R /(2 r), \epsilon$ is the dielectric constant of the fluid,

$$
\kappa^{*}=(2 r) e\left[\frac{2 c_{s} \mathrm{~N}_{\mathrm{A}}}{\epsilon \mathrm{k} T}\right]^{1 / 2}
$$

where $e$ is the charge of an electron, $c_{s}$ is the molarity of the solution, $\mathrm{N}_{\mathrm{A}}$ is Avogadro's number, and $A_{H}$ is the Hamaker constant. Combination of Eqs. (15) and (17) gives:

$$
\frac{d C}{d t}=-\frac{8 \pi D r C^{2}}{W}
$$

Thus, by Eq. (20), a large stability ratio ( $W \gg 1)$ indicates a small Brownian coagulation rate. Finally, for the more general case of the successful collision of a $j$-cluster with an $i$-cluster, the rate expression [Eq. (20)] becomes:

$$
\frac{d C_{i}}{d t}=-\frac{8 \pi \bar{D} \bar{r}_{i} C_{i} C_{j}}{W}
$$

which is a balance for $i$-clusters. The effective Brownian diffusion coefficient, $\bar{D}$, and average size, $\bar{r}$, in Eq. (21) are

$$
\bar{D}=\left(D_{i}+D_{j}\right) / 2 \quad \text { and } \bar{r}=\left(r_{i}+r_{j}\right) / 2
$$

Combination of Eqs. (16) and (22) gives:

$$
\bar{D}=\frac{\mathrm{k} T}{6 \pi \eta_{0}}\left(\frac{1}{r_{i}}+\frac{1}{r_{j}}\right)
$$

so that Eq. (21) becomes:

$$
\frac{d C_{i}}{d t}=\frac{-4 \mathrm{k} T}{3 \eta_{0} \omega}\left(\frac{1}{r_{i}}+\frac{1}{r_{j}}\right)\left(r_{i}+r_{j}\right) C_{i} C_{j} .
$$

Equation (24) describes the change in concentration of $i$-clusters by Brownian coagulation due to the presence of $j$-clusters.

Brownian "declustering" is a related process by which an $i$-cluster breaks into smaller clusters due to Brownian motion of the particles from which it is constituted. ${ }^{30,31}$ A successful declustering event will depend both on the size of the cluster (since the magnitude of Brownian forces depends on particle or cluster size) and on the "depth" of the potential well that inhibits two 
joined clusters from separating. This is given by Russel ${ }^{4}$ and many others as:

$$
\frac{d C_{i}}{d t}=\frac{-\mathrm{k} T}{\eta_{0} r_{i}^{2}} C_{i} \exp \left[\frac{V_{\min }-V_{\max }}{\mathrm{k} T}\right] .
$$

The difference $\left(V_{\min }-V_{\max }\right)$ corresponds to the difference in potential between the maximum and minimum values. A large difference, then, leads to a large negative argument on the exponential term of Eq. (25). Thus, a deep potential well implies a small rate of Brownian declustering, as the right-hand side of Eq. (25) becomes small. Finally, as in Eqs. (12) and (14), a combinatorial prefactor must be included in Eq. (25), in order to account for all of the ways by which the particles of a cluster can be subdivided. The resulting birth and death functions are

$$
B_{3, k}=\sum_{m=1}^{N_{s}} \frac{(m)(m !)}{(k !)(m-k) !} \frac{\mathrm{k} T}{\eta_{0} r_{m}^{2}} C_{m} \exp \left[\frac{V_{\min }-V_{\max }}{\mathrm{k} T}\right]
$$

and

$$
D_{3, k}=\sum_{m=k+1}^{[k / 2]} \frac{(m)(m !)}{(k !)(m-k) !} \frac{\mathrm{k} T}{\eta_{0} r_{m}^{2}} C_{m} \exp \left[\frac{V_{\min }-V_{\max }}{\mathrm{k} T}\right]
$$

\section{E. Population balance-local}

For a given slab at height $z$ in the sedimentation column, the time rate-of-change of $k$-clusters may be found by combining Eqs. (1), (8), (10), (11), (12), (14), (26), and (27) (along with auxiliary equations) to obtain:

$$
\begin{gathered}
\frac{d C_{k}}{d t}=J_{c, k}+J_{D, k}+\left[B_{1, k}+B_{2, k}+B_{3, k}\right] \\
+\left[D_{1, k}+D_{2, k}+D_{3, k}\right]
\end{gathered}
$$

where $C_{k}$ is a function of both position, $z$, and time, $t$.

\section{F. Population balance-global}

Equation (28) applies to only one cluster size in one slab. The balances are affected, however, by the population of $k$-clusters in neighboring slabs through the sedimentation term $\left(J_{c, k}\right)$ and diffusion term $\left(J_{D, k}\right)$. Further, the population of $k$-clusters in a given slab is affected by the concentration of $m$-clusters, where $m \neq k$, in the same slab through the birth and death functions ( $B_{-, k}$ and $D_{-, k}$, respectively). The equations are all of the form of Eq. (28), and they must be solved simultaneously. The number of equations involved depends upon two parameters: the number of slabs into which the cell is divided, $N_{z}$, and the maximum cluster size allowed, $N_{s}$. The resulting set of equations is of the form:

$$
\frac{\partial C_{j}}{\partial t}=f\left(C_{1}, C_{2}, \ldots, C_{N s}, z, t\right), \quad 1 \leqslant j \leqslant N,
$$

where the total number of equations to be solved is $N=\left(N_{s}\right)\left(N_{z}\right)$.

\section{G. Method of numerical solution}

Equations (29) form a set of coupled, nonlinear partial differential equations, for which analytical solutions are not available. The method of solution, then, involved a common technique ${ }^{33}$ by which the right-hand side of Eq. (29) is discretized in $z$ and held constant in $t$. A set of decoupled, ordinary differential equations results. These equations are then amenable to solution by a Runge-Kutta algorithm. ${ }^{33}$ The value of $C_{j}$ is then updated in time, for all $j$. The functions of $f$ are then updated using the new values of $C_{j}$, and the integration to the next time-step is executed. Initial conditions may be specified arbitrarily, but in this work were specified to be a uniform dispersion of one-clusters, at time $t=0$. Boundary conditions that are sufficient to accurately represent the physical nature of the process are the condition of no flux $\left[J_{c, k}=J_{D, k}=0\right.$, all $\left.k\right]$ through the top or bottom of the sedimentation cell.

\section{EXPERIMENTAL PROCEDURE}

Gamma-ray attenuation measurements were performed as a function of time and position to evaluate density evolution of alumina sediments. The apparatus directs a collimated beam $(0.32 \mathrm{~cm}$ diameter $)$ of gamma radiation from a ${ }^{137}$ cesium isotope through a plexiglass upright cylinder $(7.6 \mathrm{~cm}$ diameter) containing each suspension. Attenuation measurements were performed with a multichannel analyzer by integration of energyas-a-function-of-intensity data at the primary $662 \mathrm{keV}$ $\left({ }^{137}\right.$ cesium) peak. The experimental configuration is shown in Fig. 6. Average densities were determined by simultaneous solution of mass conservation and Beer-Lambert law equations. Details of the apparatus

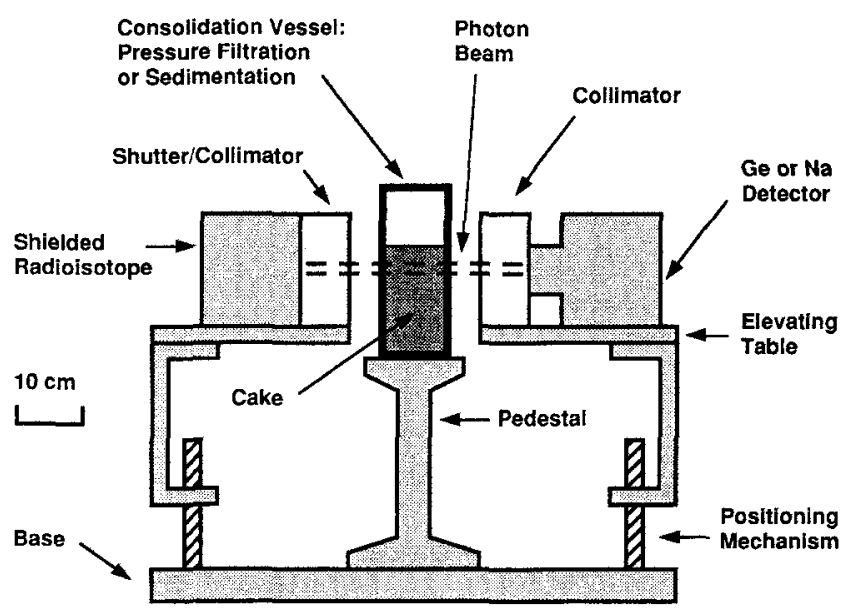

FIG. 6. Gamma-ray attenuation measurement apparatus. 
and derivations of exact equations for the alumina systems used in this study were previously disclosed. ${ }^{7}$

Colloidal suspensions were prepared using distilled water and Sumitomo type AKP-30 $\alpha-\mathrm{Al}_{2} \mathrm{O}_{3}$ powder $(0.4 \mu \mathrm{m}$ average particle size). Reagent grade $\mathrm{HCl}$ and $\mathrm{NH}_{4} \mathrm{OH}$ were added to prepare suspensions at $\mathrm{pH}=2$, $\mathrm{pH}=5$, and $\mathrm{pH}=7.3$. Suspensions were prepared using a presedimentation process to remove large agglomerates that rapidly sediment, thus causing nonuniform packing densities. Presedimentation entailed preparation of three identical suspensions with $10 \mathrm{vol} . \%$ as-received powder at $\mathrm{pH}=2$. Supernatant was siphoned from each suspension after $24 \mathrm{~h}$ of sedimentation, and $\mathrm{NH}_{4} \mathrm{OH}$ was subsequently added to two of the supernatants to raise the $\mathrm{pH}$ to 5.0 and 7.3. Gammaray attenuation experiments were subsequently performed with $500 \mathrm{~mL}$ of each supernatant. Supernatants at $\mathrm{pH}=2, \mathrm{pH}=5$, and $\mathrm{pH}=7.3$ contained, respectively, average concentrations of $5.4,4.7$, and $4.7 \mathrm{vol} . \%$ alumina, based on pycnometer measurements. Visual observations of sediment heights were recorded and correlated with the position of the collimated radiation beam.

\section{RESULTS AND DISCUSSION}

Gamma-ray attenuation measurements and computer experiments were conducted for three values of zetapotential: high, intermediate, and low. These correspond to $\mathrm{pH}$ values of 2,5 , and 7.3 , respectively. In each case, the computer experiments were conducted to account for such differences in interaction potential, as well as each of the other mechanisms described in Sec. II.

Results for sedimentation under high zeta-potential conditions $(\mathrm{pH}=2)$ are shown in Fig. 7. Under this condition, the interparticle interaction potential would be

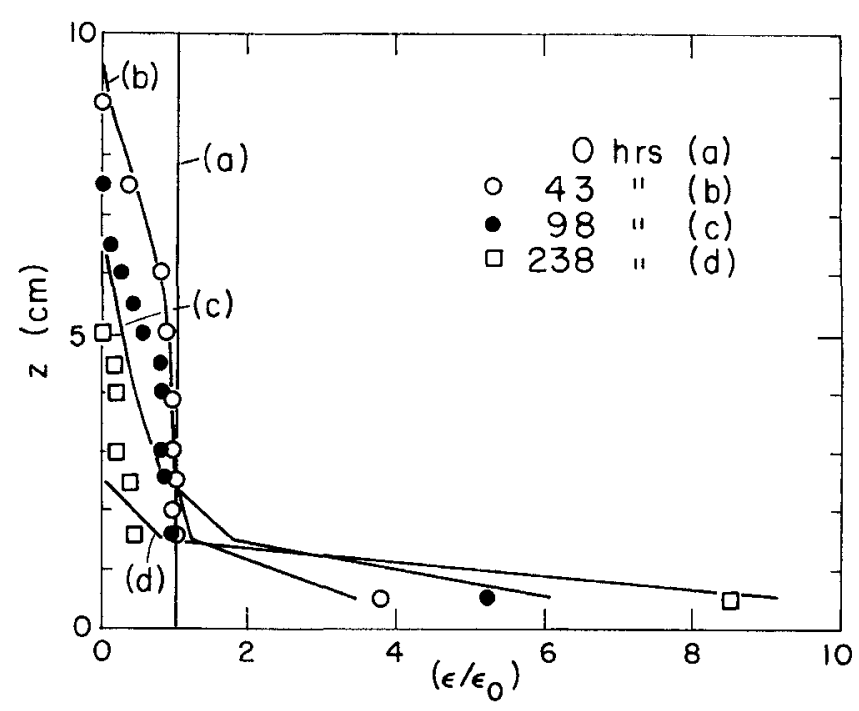

FIG. 7. Sedimentation for the high zeta potential case $(\mathrm{pH}=2)$ : solids volume fraction versus axial position, at several times. expected to be strongly repulsive, so that the suspensions could be considered to be well-dispersed. The plot depicts the solids volume fraction $\left(\boldsymbol{\epsilon} / \boldsymbol{\epsilon}_{0}\right)$ at numerous positions in the sedimentation column $(z)$, at several times during the experiment. Note that the local volume fraction is normalized with respect to the initial solids volume fraction, $\epsilon_{0}$. The experimental values of local solids volume fraction at $0,43,98$, and $238 \mathrm{~h}$ indicate that the sedimentation proceeded in a manner that is characteristic of well-dispersed suspensions: simultaneous formation of a sediment layer and clarification of the upper portion of the supernatant. The predicted solids volume fraction profiles are represented by the corresponding curves in Fig. 7. It may be seen that at early times the results of the computer experiments match those obtained in the sedimentation cell. At later times, the predictions match the experimental results in the lower portion of the cell (i.e., where $\epsilon$ has its highest values for $t>0$ ). The solids volume fraction in the upper, more dilute, portion of the cell matches less well, but still fairly accurately for this case of a well-dispersed suspension.

The evolution of cluster number density with time for the same well-dispersed $(\mathrm{pH}=2)$ suspension is shown in Fig. 8. Specifically, it shows the cluster number density for the lowermost slab in the sedimentation cell. Cluster number density is defined here for each $j$-cluster as the number of $j$-clusters per unit volume. Several features may be observed. First, it may be seen that for the well-dispersed case most of the clusters exist as one-clusters, and that the larger particle clusters have a number density several orders of magnitude lower than that of the one-clusters. This is as one would expect for a suspension containing particles that possess

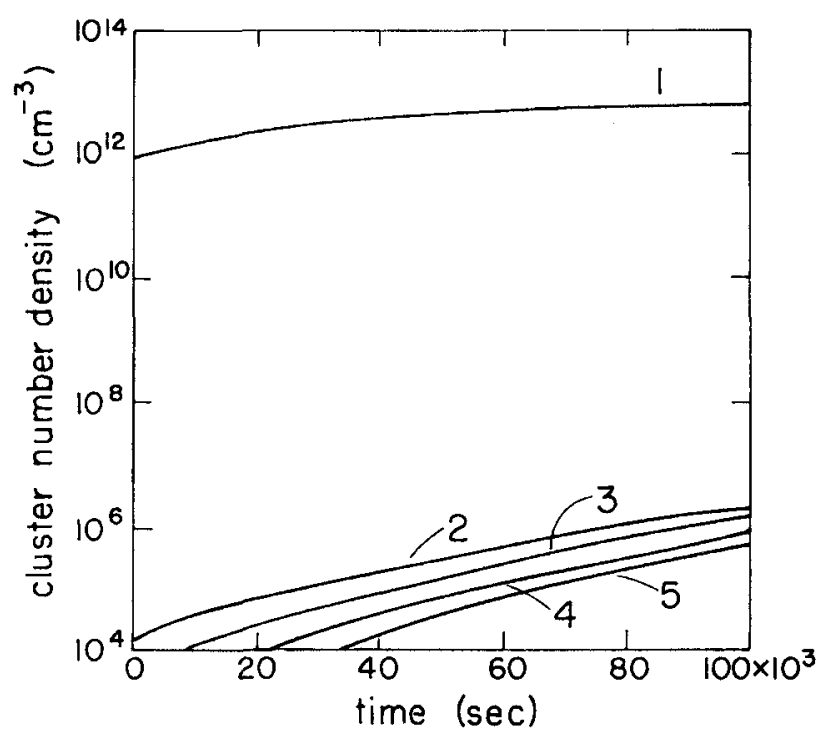

FIG. 8. Cluster number density in lowermost slab versus time, for the high zeta potential case $(\mathrm{pH}=2)$. 
a high-zeta potential. Second, it should be noted that the number density of each $j$-cluster increases with time. This is consistent with Fig. 7, in which the total solids volume fraction (that is, the sum of the volume of each $j$-cluster subpopulation) in the bottom slab increases with time. And third, the number density of $j$-clusters for $j>1$ increases more rapidly than for one-clusters, indicating that the net effect of the various coagulation and decoagulation processes is toward the increase in number density of $2^{+}$-clusters.

Results for sedimentation under moderate zeta potential conditions $(\mathrm{pH}=5)$ are shown in Fig. 9. It may be seen from the experimental points that sedimentation again proceeded as for a dispersed case: decreasing and increasing solids volume fractions in the uppermost and lowermost slabs, respectively. The key feature in Fig. 9, however, is the agreement between predicted and experimental values, in most slabs at all times reported. This is likely due to the fact that the balance of coagulation and decoagulation mechanisms was properly accounted for and that the net effect of all contributions properly predicted the evolution of the solids volume fraction with time. Figure 10, which shows the cluster number density for the $\mathrm{pH}=5$ suspension, also supports this observation. The number density of all $2^{+}$-clusters is significantly greater than that shown in Fig. 8. This is consistent with the expected outcome of the sedimentation of particles that possess a smaller zeta potential: the stability ratio, as defined by Eq. (17), increases and the number of successful collisions would therefore increase. A correspondingly greater cluster number density for all $2^{+}$-clusters would be the result. As in Fig. 8, Fig. 10 also shows that the number density of $2^{+}$-clusters increases relatively more rapidly than for one-clusters. Again, this is likely due to the fact

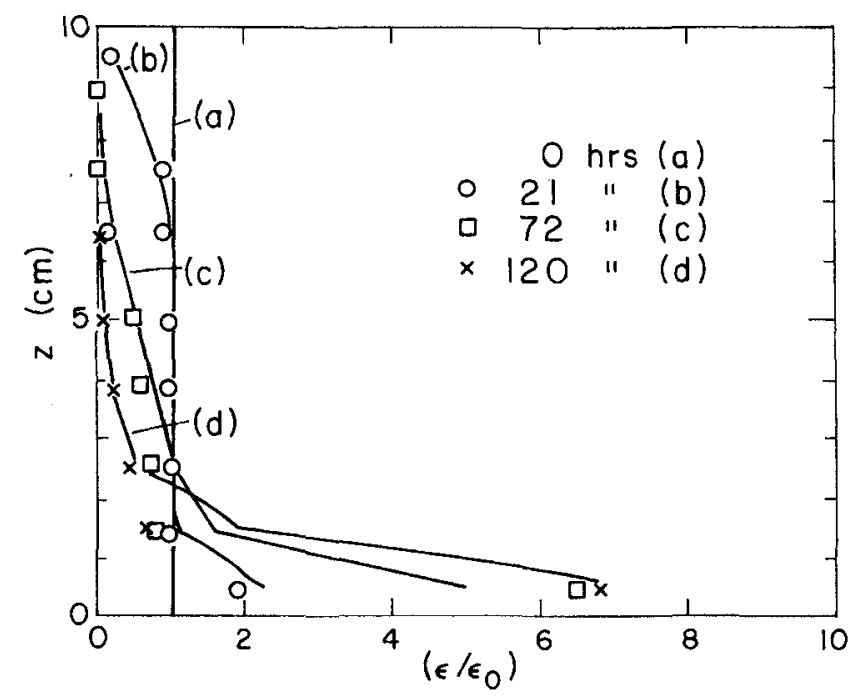

FIG. 9. Sedimentation for the intermediate zeta potential case $(\mathrm{pH}=$ 5): solids volume fraction versus axial position, at several times.

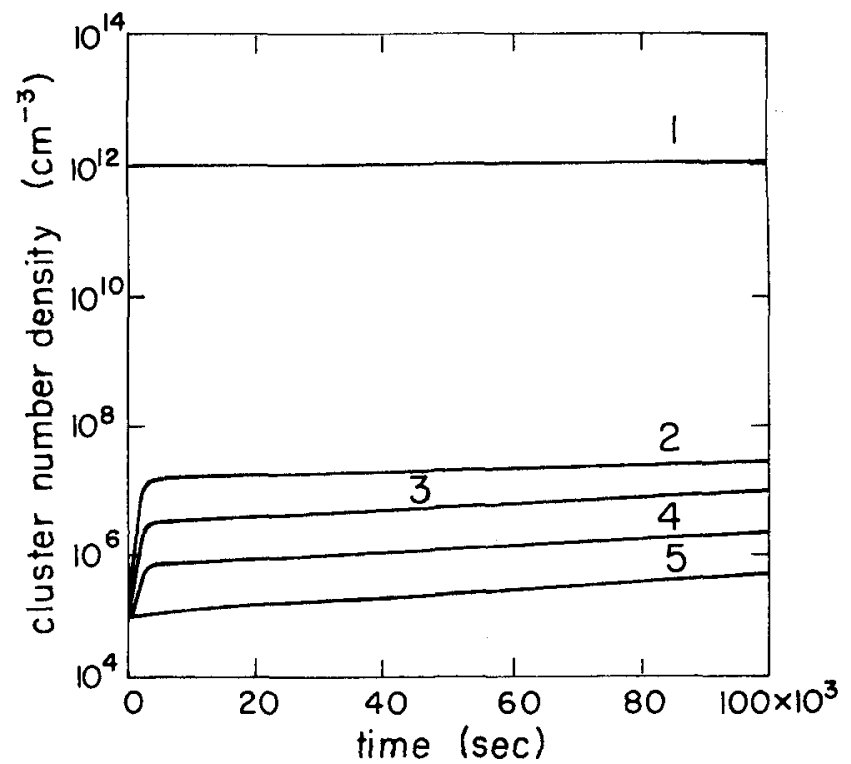

FIG. 10. Cluster number density in lowermost slab versus time, for the intermediate zeta potential case $(\mathrm{pH}=5)$.

that conditions favor coagulation over decoagulation, particularly as the interparticle potential is lower in Fig. 10 than in Fig. 8.

Results for sedimentation under relatively low zeta potential conditions $(\mathrm{pH}=7.3)$ are shown in Fig. 11. It may be observed in Fig. 11 that the sedimentation occurs somewhat more quickly here than in the two previous cases. Further, it may be seen that the experimental and predicted solids volume fraction profiles correspond quite well throughout the sedimentation process, except at later times in the lower slabs when the solids volume fraction is quite large. This again underscores the ability of the theoretical experiments to predict successfully the

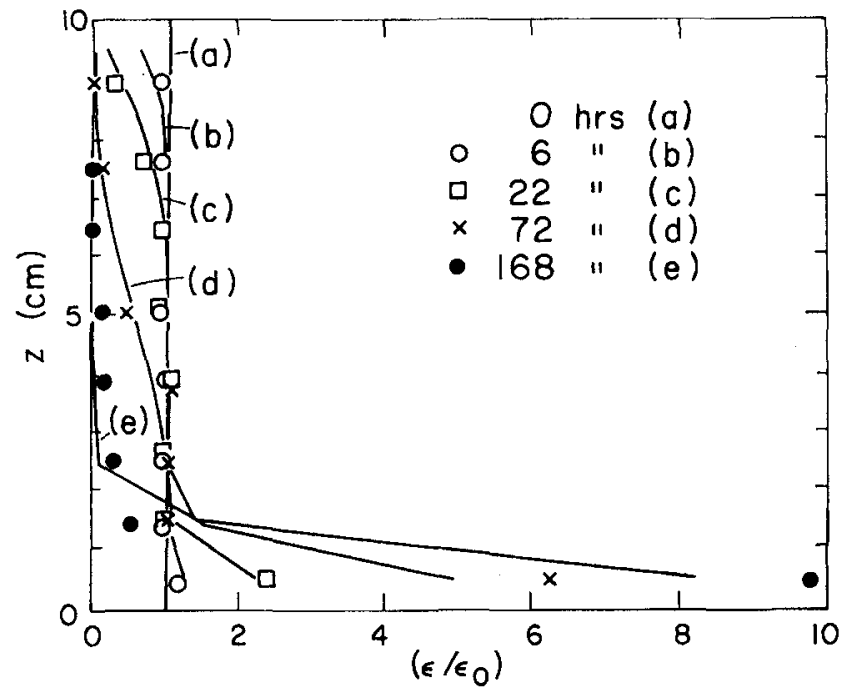

FIG. 11. Sedimentation for the low zeta potential case $(\mathrm{pH}=7.3)$ : solids volume fraction versus axial position, at several times. 
outcome of the sedimentation experiments. Figure 12, on the other hand, indicates that the trend (illustrated above in Figs. 8 and 10) toward increased cluster number density with decreasing interparticle potential is continued for the low zeta potential case. Again, clusters form quickly at early times, and increase in number density more gradually at later times. The primary difference between this and the earlier cases is that the cluster number density for $j \geqslant 2$ is several orders of magnitude greater in the present case $(\mathrm{pH}=7.3)$. Furthermore, as before, the rate of number density increase of $2^{+}$-clusters is greater than that for one-clusters.

\section{CONCLUSIONS}

This paper describes the results of a combined theoretical and experimental study of the sedimentation of a flocculating suspension. In the theoretical portion, a population balance model was used successfully to describe sedimentation in a particle-containing suspension in which the particles can form long-lived clusters. The model explicitly included several physicochemical processes that are known to significantly alter a simple Stokesian description of sedimentation: diffusion, hindered settling, hydrodynamic clustering and declustering, and Brownian clustering and declustering. In the experimental portion, gamma-ray densitometry was used to measure the solids volume fraction of a ceramic particle-containing suspension, as a function of both time and position. The suspensions contained submicron $\mathrm{Al}_{2} \mathrm{O}_{3}$ powders, and each was prepared in order to control precisely interparticle interaction potential, and thus the tendency of the particles to flocculate.

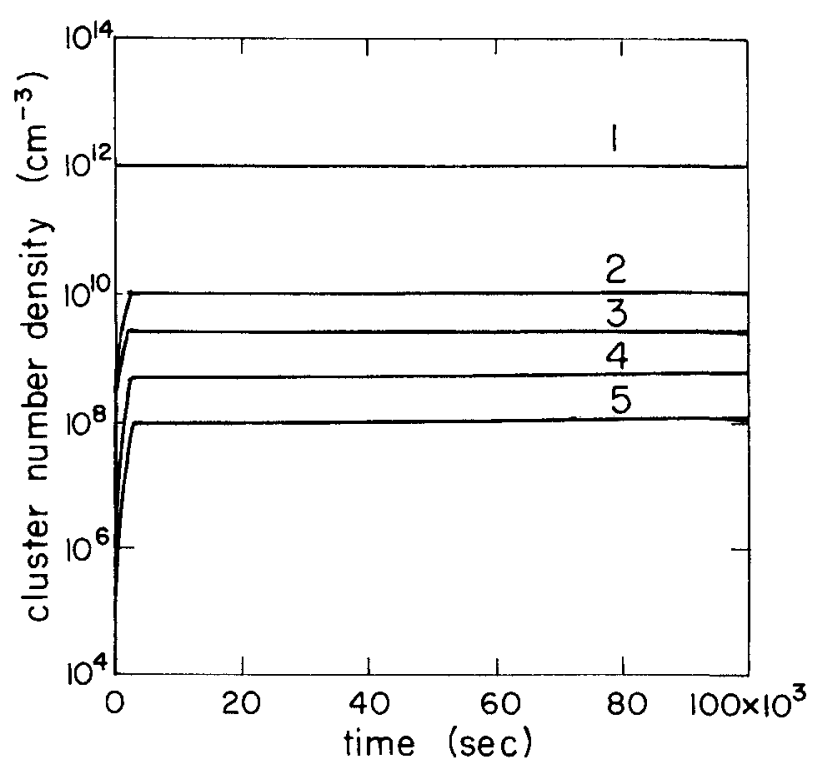

FIG. 12. Cluster number density in lowermost slab versus time, for the low zeta potential case $(\mathrm{pH}=7.3)$.
Experimental measurements were then compared with model predictions in order to verify the results of the theoretical calculations. Very good agreement was observed between experimental and theoretical results, particularly in all regions above the sediment layer. The successful results obtained in the present study indicate that the population balance model can be extended to describe, for example, centrifugation, slip-casting, pressure filtration, and sedimentation in mixed particle suspensions.

\section{REFERENCES}

1. J. S. Reed, Introduction to Ceramic Engineering (John Wiley, New York, 1988).

2. F. M. Auzerais, R. Jackson, W. B. Russel, and W.F. Murphy, J. Fluid Mech. 221, 613-639 (1990).

3. M. A. Al-Naafa and M.S. Selim, Can. J. Chem. Eng. 67, 253-264 (1989).

4. W. B. Russel, The Dynamics of Colloidal Systems (University of Wisconsin Press, Madison, WI, 1987).

5. F. F. Lange, J. Am. Ceram. Soc. 72 (1), 3-15 (1989).

6. C. H. Schilling, W-H. Shih, and I. A. Aksay, in Ceramic Powder Science $I V$, edited by S. Hiranno, G. L. Messing, and H. Hausner (American Ceramic Society, Westerville, OH, 1991), Vol. 22, pp. 307-321.

7. L. Bergström, C.H. Schilling, and I. A. Aksay, J. Am. Ceram. Soc. (1993, in press).

8. G. K. Batchelor, J. Fluid Mech. 52 (2), 245-268 (1972).

9. R.H. Davis and K.H. Birdsell, A.I.Ch.E.J. 34 (1), 123-129 (1988).

10. Y.T. Shih, D. Gidaspow, and D. T. Wasan, Powder Technol. 50, 201-215 (1987).

11. G. K. Batchelor, J. Fluid Mech. 119, 379-408 (1982).

12. G. K. Batchelor and C-S. Wen, J. Fluid Mech. 124, 495-528 (1982).

13. P. Somasundaran, Fine Particles Processing, edited by P. Somasundaran (American Institute of Mining, Metallurgical, and Petroleum Engineers, Inc., New York, 1980), pp. 947-976.

14. A. Suzuki and I. Kashiki, Ind. Eng. Chem. Res. 26, 1464-1548 (1987).

15. S. G. Mason, J. Colloid Interf. Sci. 58 (2), 275-285 (1977).

16. R. D. Harding, J. Colloid Interf. Sci. 40 (2), 164-173 (1972).

17. G.C. Jeffery and R.H. Ottewill, Colloid Polym. Sci. 268, 179-189 (1990).

18. Z. Xu and R-H. Yoon, J. Colloid Interf. Sci. 134 (2), 427-434 (1990).

19. K. E. Davis, W. B. Russel, and W. J. Glantschnig, J. Chem. Soc. Faraday Trans. 83 (3), 411-424 (1991).

20. F.M. Auzerais, R. Jackson, and W.B. Russel, J. Fluid Mech. 195, 437-462 (1988).

21. R. Buscall, Colloid Surf. 43, 33-53 (1990).

22. C.H. Schilling and I. A. Aksay, Transactions of the Canadian University-Industry Council on Advanced Ceramics-Third Workshop, February 24-25, 1987, Montreal, Quebec.

23. R. B. Bird, W. E. Stewart, and E. N. Lightfoot, Transport Phenomena (John Wiley, New York, 1960).

24. P.T. L. Koh, J. R. G. Andrews, and P. H. T. Uhlherr, Chem. Eng. Sci. 42 (2), 353-362 (1987).

25. G. F. Froment and K. B. Bischoff, Chemical Reactor Analysis and Design (John Wiley, New York, 1979).

26. J. H. Clarke, A. N. Clarke, and D. J. Wilson, Sep. Sci. Tech. 13, $767-789$ (1978). 
27. R. D. Vigil and R. M. Ziff, J. Colloid Interf. Sci. 133 (1), 257-264 (1989).

28. G. K. Batchelor and J. T. Green, J. Fluid Mech. 56 (3), 401-427 (1972).

29. C. W. J. Beenakker and P. Mazur, Phys. Lett. 91 (6), 290-291 (1982).

30. I. A. Valioulis and E. J. List, Adv. Colloid Interf. Sci. 20, 1-20 (1984).
31. G. D. Parfitt and J. Peacock, Surf. Colloid Sci. 10, 163-226 (1978).

32. A. Molski, Colloid Polym. Sci. 267, 371-375 (1989).

33. B. Carnahan, H. A. Luther, and J. O. Wilkes, Applied Numerical Methods (John Wiley, New York, 1969). 